Cahiers

d'ethnomusicologie

\section{Cahiers d'ethnomusicologie}

Anciennement Cahiers de musiques traditionnelles

8 | 1995

Terrains

\title{
Calabre. Musiques de fêtes
}

1993

\section{Antonello Ricci}

Traducteur : Georges Goormaghtigh

\section{OpenEdition}

\section{Journals}

Édition électronique

URL : http://journals.openedition.org/ethnomusicologie/1246

ISSN : 2235-7688

Éditeur

ADEM - Ateliers d'ethnomusicologie

Édition imprimée

Date de publication : 31 décembre 1995

Pagination : 252-254

ISBN : 2-8257-0537-3

ISSN : $1662-372 X$

Référence électronique

Antonello Ricci, « Calabre. Musiques de fêtes », Cahiers d'ethnomusicologie [En ligne], 8| 1995, mis en ligne le 04 janvier 2012, consulté le 19 avril 2019. URL : http://journals.openedition.org/ ethnomusicologie/1246

Ce document a été généré automatiquement le 19 avril 2019.

Tous droits réservés 


\title{
Calabre. Musiques de fêtes
}

1993

\author{
Antonello Ricci
}

Traduction : Georges Goormaghtigh

\section{RÉFÉRENCE}

Calabre. Musiques de fêtes.Enregistrements, choix des plages et notice de Goffredo Plastino. 1 CD INEDIT W 260051, 1993.

\section{NOTE DE L'ÉDITEUR}

Traduit de l'italien par Georges Goormaghtigh.

1 La Calabre, région la plus méridionale de l'Italie continentale, possède, aujourd'hui encore, un patrimoine musical populaire des plus riches et des plus élaborés. C'est probablement pour cette raison que de nombreux chercheurs, pas uniquement italiens, se sont intéressés et s'intéressent encore à la culture musicale de cette région extrême de la péninsule.

Dernier en date de la vaste discographie concernant la Calabre, ce CD, réalisé par Goffredo Plastino, un ethnomusicologue élève de Diego Carpitella, est consacré à la musique et aux phénomènes paramusicaux rencontrés au cours du déroulement de plusieurs fêtes religieuses populaires. Pendant les périodes festives, en particulier celles relatives aux pèlerinages et à la permanence près des sanctuaires, le besoin de s'exprimer à travers un code sonore et musical correspond à une nécessité interne de la communauté et met en évidence la fonction sociale du chant et de la musique paysanne du sud de l'Italie. A côté des répertoires formalisés (vocaux et instrumentaux), un aspect particulièrement intéressant de ce disque est l'attention portée à la "paramusique ", c'est-à-dire à tous les phénomènes sonores qui ne sont pas strictement formalisés dans un langage distinctement musical: sons de différents idiophones, cris, invocations etc. Un tel 
ensemble de formes expressives n'est pas mis en œuvre de façon accidentelle, mais constitue un réseau serré de communication et de représentations qui imprègne la dimension orale/auriculaire dont le système festif est le véhicule privilégié.

3 L'anthologie est constituée de vingt-deux morceaux enregistrés sur le terrain au cours de ces dix dernières années, dans des localités appartenant aux trois provinces calabraises. Certaines des principales fêtes religieuses à caractère régional y sont illustrées, ainsi que d'autres d'intérêt plus local (dont quelques unes étaient inconnues à ce jour), sans oublier les fêtes importantes du calendrier (Noël, Pâques), le tout caractérisé par des ambiances sonores toujours précises et significatives.

4 La succession des plages se présente selon un ordre qui suit le cycle de l'année. Les cinq premiers enregistrements appartiennent à la période hivernale. On commence avec un Noël à la cornemuse zampogna, auquel succède une synthèse musicale de la fête de San Leone à Saracena (province de Cosenza). Signalons, dans cette dernière sélection, la présence d'idiophones jusqu'alors inconnus, les nimule. Viennent ensuite plusieurs enregistrements, effectués au printemps, qui se rattachent aux rituels de la Passion du Christ, puis la longue et riche séquence estivale qui débute avec des documents enregistrés à Seminara (province de Reggio di Calabria) au cours de la fête de la Vierge des pauvres : la Vierge noire en l'honneur de laquelle, au rythme des tambourins, on fait danser les "géants", deux grandes marionnettes de papier mâché animées par deux danseurs-porteurs. A Riace (province de Reggio di Calabria), après diverses tarentelles, prières chantées et rosaires, la séquence estivale s'achève sur la fête des saints Cosme et Damien remarquable par la présence massive de musiciens et danseurs tziganes qui s'y rassemblent pour l'occasion. Le disque se termine sur une autre plage de musique de Noël pour zampogna qui clôt symboliquement le cycle de l'année. La plaquette qui accompagne le $\mathrm{CD}$ constitue un bon guide pour l'écoute et une bonne introduction au monde musical et sonore des fêtes enregistrées. L'auteur a eu soin de préciser le rôle des répertoires religieux liés à des fonctions de dévotion spécifiques, le moment et les lieux destinés à leur exécution, ainsi que de décrire les instruments de musique utilisés et de fournir une documentation sur les différentes fêtes. Enfin, les textes des chants enregistrés sont transcrits.

5 Ce $\mathrm{CD}$ a été réalisé dans une optique originale et inédite : il présente la partie du répertoire musical calabrais qui s'exprime à l'occasion de moments rituels et dévotionnels. Il met ainsi en lumière la vitalité qui anime aujourd'hui l'expression sonore et musicale d'une grande partie de la population de cette région malgré les inévitables changements culturels. En même temps, grâce à la réalisation d'enregistrements d'une bonne qualité technique, l'auteur permet à l'auditeur de s'immerger dans l'atmosphère sonore des différents moments de la fête, car «la sonorité d'ensemble de la fête est une caractéristique culturelle tout aussi importante que les sons ou les musiques qui peuvent être produits lors de son déroulement. » Un seul petit reproche concernant le choix de l'image de couverture: une illustration $\mathrm{du} \mathrm{xVII}^{\mathrm{e}}$ siècle -fort belle au demeurant- qui confère au disque une saveur antique en contradiction avec ce qui semblerait être le propos de l'auteur : apporter un témoignage sur une réalité d'aujourd'hui, en la restituant avec des enregistrements digitaux. 